Linguistique, littérature, didactique

141-142 | 2009

La synonymie

\title{
Arriver et venir - quand la deixis fait (et ne fait pas) la différence
}

Dorota Sikora

\section{(2) OpenEdition \\ 1 Journals}

\section{Édition électronique}

URL : https://journals.openedition.org/pratiques/1324

DOI : 10.4000/pratiques.1324

ISSN : 2425-2042

Éditeur

Centre de recherche sur les médiations (CREM)

\section{Édition imprimée}

Date de publication : 15 juin 2009

Pagination : 138-149

\section{Référence électronique}

Dorota Sikora, "Arriver et venir - quand la deixis fait (et ne fait pas) la différence », Pratiques [En ligne], 141-142 | 2009, mis en ligne le 20 juin 2014, consulté le 10 mars 2023. URL : http://

journals.openedition.org/pratiques/1324; DOI : https://doi.org/10.4000/pratiques.1324

Tous droits réservés 


\section{Arriver et venir - quand la deixis fait (et ne fait pas) la différence}

\section{Dorota Sikora}

ATILF - CNRS (UMR 7118) - Nancy - Université

\section{Introduction}

Venir et arriver, arriver et venir : est-ce bonnet blanc et blanc bonnet ? Non - répondra certainement plus d'un étudiant de français langue étrangère, repris plusieurs fois pour avoir employé l'un pour l'autre, là où il ne fallait surtout pas le faire. Oui - répliquera un autre, se souvenant d'un exercice dans lequel il devait, avec le souci légitime d'élargir son vocabulaire, " remplacer le mot entre parenthèses par son synonyme ». Dans cet article, nous nous proposons d'observer les relations synonymiques qui s'établissent entre ces deux verbes dans leurs emplois spatiaux, c'est-à-dire ceux qui réfèrent à des situations de mouvement. Il convient donc de préciser ce que nous entendons par mouvement ${ }^{(1)}$.

\section{La notion de mouvement (déplacement)}

En suivant Asher et Sablayrolles (1995: 166), nous admettons que « [L]e mouvement lui-même est à chaque fois un type d'événement et il consiste dans un changement de position spatiale à travers le temps ${ }^{(2)}$. Talmy (2000/II : 25) définit les quatre éléments conceptuels qui forment une situation ou un événement de mouvement. Figure est un objet mobile (ou bien conceptualisé comme tel, et c'est ainsi que nous employons ce terme). Site (Ground) est un point de référence par rapport auquel le mouvement de figure peut être décrit et il recouvre, selon le cas, la source (lieu initial) ou bien le terminus (lieu final) du déplacement. L'ensemble des positions adoptées par figure dans le cours de son déplacement forme sa trajectoire. Dans cet article, nous allons donc employer le terme de mouvement comme synonyme de déplacement.

(1) Certains auteurs intègrent dans cette classe de sens (Asnès et Kupferman 2008) des verbes comme dévisser, enlever, etc. (cf. Boons, 1985, 1987).

(2) Asher et Sablayrolles (1995: 166): «Motion is a property of objects (i.e. objects move), but motions themselves are a type of event. Motions are changes of some sort of spatial position over time $»$. 


\section{Qu'en est-il de la synonymie entre arriver et venir ?}

\subsection{Quelques remarques sur la synonymie "en général »}

Depuis Duchacek (1979), un accord semble établi sur la notion de synonymie : sont synonymes « les unités lexicales de sens identique ou presque identique ou du moins aussi proches de sens qu'elles sont interchangeables dans certains contextes » (Duchacek, 1979 : 9). C'est aussi à Duchacek que nous devons l'idée selon laquelle il existe des degrés de synonymie allant de l'identité totale (absolue) à une quasi-identité de sens ou synonymie partielle plus ou moins forte.

La distinction généralement opérée entre la synonymie totale (ou absolue) et la quasi-synonymie est le reflet du caractère dynamique des relations synonymiques. La synonymie totale (absolue) suppose une identité « parfaite » de sens entre les expressions A et B. Elles sont alors substituables dans tous les contextes grammaticalement bien formés : là où A est approprié, B l'est aussi et vice versa. De tels cas de synonymie sont extrêmement rares, sinon inexistants. Le concept de synonymie absolue sert plutôt de point de référence cognitive permettant de considérer la synonymie comme un phénomène scalaire, plus ou moins éloigné de ce cas idéal.

D'un autre côté, la synonymie partielle recouvre une quantité de relations intuitivement perçues comme celles de ressemblance, mais différant en réalité d'un point de vue tant sémantique (degrés de prototypicalité variés, différences aspectuelles entre les lexèmes concernés) que stylistique (Duchacek 1979, Cruse 2004).

Si l'étude de Duchacek (1979) constitue, depuis trente ans, un point de référence, voire un point de départ, théorique et terminologique pour de nombreuses réflexions sur la synonymie, la problématique n'en reste pas moins riche et stimulante. Il ne saurait en être autrement dans la mesure où les relations plus ou moins synonymiques entre deux unités lexicales ont un caractère dynamique : l'identité ressentie par les locuteurs n'est pas une constante. L'expérience d'un étudiant de FLE, quelque peu perdu face aux relations synonymiques instables entre les occurrences de venir et celles d'arriver, le montre bien. Elles s'établissent dans certains emplois, elles disparaissent lorsque le co-texte, voire le contexte, change. Cette instabilité rend leur acquisition particulièrement difficile.

Mais c'est grâce à ce caractère dynamique et fluctuant que la synonymie ne manque pas de sel. "Si nous voyons dans la synonymie simplement une identité de sens, elle apparaîtra comme une relation somme toute peu intéressante. Si nous considérons au contraire que les synonymes sont des mots dont les ressemblances sémantiques sont plus saillantes que les différences susceptibles de les opposer, une vaste sphère d'investigation s'ouvre. Quel est le type de différences qui ne met pas en cause 1'intuition d'identité ? Comment se fait-il que les synonymes soient si fréquents ? (Alors qu'une identité absolue ne semble pas motivée d'un point de vue fonctionnel) Existe-t-il des domaines du lexique où ils sont particulièrement nombreux ?» ${ }^{(3)}$ (Cruse $\left.2004: 154\right)$.

(3) «If we interpret synonymy simply as sameness of meaning, then it would appear to be a rather uninteresting relation; if, however, we say that synonyms are words whose semantic similarities are more salient than their differences, then a potential area of interest opens up. What sort of difference do not distroy an intuition of sameness? Why are such synonyms so frequent? (Absolute sameness of meaning would seem to be funcionnally unmotivated). Do they proliferate in particular areas of the vocabulary ? » (Cruse $2004: 154$ ). 


\subsection{Synonymie dans un cas spécifique : arriver et venir en tant que quasi-synonymes en langue}

Arriver et venir, sont-ils réellement des synonymes ? Deux réponses semblent s'esquisser selon que l'on s'en tienne aux définitions lexicographiques ou que l'on s'en remette aux faits linguistiques, tels que le test de substituabilité. En effet, les emplois synonymiques de arriver et de venir sont largement confirmés : parmi les soixante expressions répertoriées par DicoSyn ${ }^{(4)}$ comme plus ou moins équivalentes de venir, arriver vient en troisième position. Inversement, venir n'est que quatrième sur la liste des cinquante quatre synonymes possibles d'arriver ${ }^{(5)}$.

Si l'on s'en tient à des définitions lexicographiques qui, par leur nature, sanctionnent ce qu'offre la langue, l'éventuelle synonymie de venir et de arriver devrait paraître plutôt incertaine.

\subsubsection{Arriver}

Le Trésor de la Langue Française informatisé(désormais TLFi) précise que les emplois spatiaux de ce verbe ne montrent aujourd'hui aucune préférence étymologique pour les déplacements sur l'eau. Arriver signifie " parvenir à l'étape fixée, parvenir à atteindre (qqn) après des difficultés, parvenir à destination » ${ }^{(6)}$. Par ailleurs, la destination (terminus dans la terminologie que nous avons adoptée) peut être explicitement spécifiée par un complément dit de lieu. Arriver apparaît également dans des emplois absolus, c'est-à-dire sans que le terminus soit clairement identifié par un complément.

\subsubsection{Venir}

La définition du/des sens spatiaux de venir présente une structure différente, selon que le verbe est suivi ou non d'un complément de lieu. Construit avec un complément de lieu, le verbe signifie « se déplacer dans la direction d'un lieu ou d'une personne de référence ${ }^{(7)}$. Deux périphrases sont proposées pour les constructions dans lesquelles venir est accompagné d'un "complément de lieu indiquant le terme du déplacement » : a. " aller auprès d'une personne, dans un lieu, pris comme référence », comme dans venir à l'école et b. «aller, se diriger vers », par exemple dans des constructions telles que venir au devant de quelqu'un. Si dans les deux cas le terminus est explicitement nommé, conformément à la définition b., il n'est pas forcément atteint par figure.

Outre les ressemblances que l'on repère dans les constructions grammaticales des deux verbes, les définitions du TLFi mettent en avant des points importants pour notre discussion des relations synonymiques entre arriver et venir. Si venir peut être traité comme un verbe de déplacement dans la mesure où il réfère à un événement de mouvement (figure change d'emplacement, en adoptant des localisations successives par rapport au terminus), arriver semble se focaliser non pas sur le déplacement, mais sur la mise en relation de figure et du terminus. En simplifiant, les deux verbes ne réfèrent donc pas à la même chose.

(4) http ://www.cnrtl.fr/synonymie/venir/verbe, http://www.cnrtl.fr/synonymie/arriver

(5) Il nous est impossible, faute de place, de discuter cette asymétrie des rapports synonymiques entre arriver et venir. Il nous semble cependant qu'une des raisons possibles serait à chercher parmi les contraintes pesant sur la nature de l'argument sujet de venir. Arriver en est dépourvu et accepte bien des arguments non agentifs.

(6) http ://atilf.atilf.fr/dendien/scripts/tlfiv5/advanced.exe ?8; $\mathrm{s}=2172557160$

(7) http ://atilf.atilf.fr/dendien/scripts/tlfiv5/visusel.exe ?12;s=117020265; ; =1; nat = ;sol=3 
Cependant, les données fournies par DicoSyn indiquent un lien synonymique fort entre les deux. A notre avis, il s'explique par une quasi-synonymie reposant sur un rapport métonymique de type partie-tout entre les sens de arriver et de venir, le premier désignant la phase finale d'un déplacement.

\section{3. «...lieu ou personne pris comme référence " ou le caractère déictique de venir}

La comparaison des deux définitions permet de remarquer une autre différence de taille. Outre le sens descriptif qui fait de arriver et de venir des candidats pour une relation de quasi-synonymie, venir présente une caractéristique en plus. Nous pensons notamment aux termes de lieu ou de personne de référence. Avec ou sans complément de lieu, le terminus est identifié à travers un lieu ou une personne de référence. On constate donc qu'en dehors du sens descriptif de mouvement paraphrasable par aller, venir a aussi un sens instructionnel ou procédural permettant d'effectuer une opération d'identification de l'entité qui servira de référence. Une telle procédure ne semble pas nécessaire dans le cas de arriver.

On comprend dès lors mieux pourquoi le maniement et les rapports entre ces deux verbes présentent tant de difficultés en cours d'apprentissage de français langue étrangère. Il s'agit de comprendre la nature d'une opération dont le déroulement n'est pas simple à établir. Pour le faire, le locuteur doit suivre une certaine procédure. La question serait donc double : 1) comment trouve-t-on « le lieu ou la personne de référence » ? 2) comment se fait-il que dans certains co(n)-textes, le sens procédural n'empêche pas les relations de synonymie entre venir et arriver? Ce qui est une autre façon de s'interroger, avec Cruse (2004), sur les ressemblances plus fortes que les différences.

Afin de saisir la démarche qui conduit le locuteur vers la personne ou le lieu de référence, nous proposons deux exemples d'emplois du verbe venir :

(1) Viens !

(2) Viens au concert!

Dans (1), facilement accompagné d'un geste, l'énonciateur constitue le terminus du déplacement. Plus précisément, le lieu est identifié à travers sa position dans l'espace. Dans (2), le complément de lieu au concert identifie le lieu-terminus indépendamment de l'énonciateur. Cela ne signifie pas pour autant que l'énonciateur ne joue aucun rôle dans la signification de l'énoncé : en employant venir dans (2), il signale que lui-même compte se rendre au concert. En d'autres termes, il se situe au terminus du déplacement.

Ainsi, les exemples (1) et (2) montrent-ils les deux modes d'implication déictique de l'énonciateur :

1. le lieu de référence est identifié comme celui où se trouve l'énonciateur («lieu englobant » chez Kleiber, 2008) ${ }^{(8)}$.

2. le lieu de référence est identifié indépendamment de l'énonciateur, mais celui-ci n'est pas indifférent pour la signification de l'énoncé, puisqu'en recourant à venir, il indique sa présence au terminus du déplacement.

Ces observations confirment le caractère déictique largement reconnu de venir.

(8) Ce n'est sans doute pas un hasard si, dans ce type d'emplois, venir est suivi de ici à valeur englobante ( $c f$. Kleiber, $2008: 133$ ). 
Elles s'inscrivent, nous semble-t-il, dans les remarques de Kleiber (2008) qui propose une conception token-réflexive de la deixis :

Une expression token-réflexive est une expression qui renvoie à son référent par l'intermédiaire d'éléments spatio-temporellement reliés à son occurrence.

Kleiber, $2008: 124$

Pour la suite de notre réflexion, nous adopterons la thèse de travail selon laquelle l'énonciateur en tant qu' « élément spatio-temporellement relié » à certaines occurrences aussi bien de venir qu'à celles d'arriver joue un rôle important, voire décisif, pour les relations synonymiques (ou pour leur absence) entre ces deux verbes. En observant les possibilités de substitution dans des co(n)-textes précis, nous nous reposerons sur le classement proposé par Fuchs (1997).

\subsection{Les types de co-textes (Fuchs 1997)}

L'existence ou non des relations synonymiques entre deux lexèmes, ou plus généralement, entre deux expressions, est vérifiée à l'aide du test de substitution. Il consiste à remplacer, dans un co-texte, un lexème (une expression) par celui (celle) que l'on considère comme équivalent(e), pour vérifier si cette opération produit une modification dans sa signification. La possibilité d'une commutation témoigne de la synonymie, son impossibilité l'exclut. En observant les effets de telles substitutions dans les environnements linguistiques, Fuchs (1997) distingue deux principaux types de co-textes. Il existe, en effet, des co-textes dissimulateurs et des co-textes assimilateurs. Chacun des deux types possède une variante forte et une variante faible. Au total, quatre cas de figure doivent être envisagés :

1. un co-texte dissimulateur fort interdit toute substitution;

2. alors qu'un co-texte dissimulateur faible l'autorise, mais les deux énoncés ainsi obtenus ne sont pas des paraphrases. En d'autres termes, leurs sens sont différents et l'on ne peut pas considérer les lexèmes (ou les expressions) substitué(e)s comme synonymes.

3. Les co-textes assimilateurs faibles autorisent une substitution, mais celleci aboutit à une quasi-synonymie, c'est-à-dire à un recouvrement partiel de la signification des énoncés.

4. Les co-textes assimilateurs forts sont ceux dans lesquels une substitution produit des énoncés qui sont des paraphrases l'un de l'autre.

\section{Venir et arriver en co-texte ${ }^{(9)}$}

\section{1. ...dissimulateur fort}

Nous commençons nos analyses par des co-textes qui excluent la substitution synonymique, car repérer la nature de l'élément responsable du blocage nous semble tout aussi instructif que cerner une similitude. Observons l'exemple (3) :

(3) «-Nous avons décidé d'aller voir la tombe de papa. Viens si tu veux, il t'aimait bien ». Cette dernière phrase est ridicule mais je vais avec elles. (Frantext)

(9) Je tiens à remercier chaleureusement tous les membres de l'équipe Lexique (ATILF-CNRS UMR 7118) qui ont bien accepté de participer à l'atelier de travail du 14 mars 2008. Leurs avis sur les exemples que j'ai pu recueillir et leurs remarques m'ont été précieuses. Je suis seule responsable d'éventuelles erreurs et incompréhensions des idées exposées lors de nos discussions. 
Remplacer venir par arriver est, en effet, impossible, comme le montre (3') :

(3') «-Nous avons décidé d'aller voir la tombe de papa. *Arrive si tu veux, il t'aimait bien ». Cette dernière phrase est ridicule mais je vais avec elles.

L'ensemble de (3) forme un co-texte dissimulateur fort, mais - pourrait-on objecter - cela n'est pas lié au caractère déictique de venir. On pourrait simplement faire remarquer que arriver, de manière générale, résiste à la forme impérative, tout comme certains autres lexèmes verbaux susceptibles de fonctionner en synonymie avec lui, par exemple parvenir. Prenons donc un autre exemple :

(4) Je te donne mon disque mais tu viens à mon concert. (forum Internet)

La substitution de venir par arriver produit l'énoncé (4') qualifié de douteux par nos informateurs :

(4') ? Je te donne mon disque mais tu arrives à mon concert.

Dans (4), le co-texte dissimulateur fort ne peut pas être expliqué par 1'emploi de l'impératif, puisque venir est employé à l'indicatif.

Indépendamment de la forme impérative, dans l'exemple (3) tout comme dans (4), l'engagement de l'énonciateur empêche la substitution. Dans (3), l'énoncé contenant l'occurrence de venir est enchâssé dans un discours direct pris en charge par une énonciatrice (l'une d' « elles »), alors que la séquence du discours dans son ensemble doit être attribuée à un autre locuteur, celui qui assume le je. L'énonciatrice de venir signale sa présence au terminus, c'est-à-dire à la tombe de papa. Nous avons donc affaire au second mode d'implication déictique.

L'exemple (4) présente un cas similaire, la forme impérative en moins. En faisant recours à venir, l'énonciateur qui, cette fois est également le locuteur, signifie qu'il sera au concert, lieu de référence, terminus du déplacement. On pourrait, certes, objecter que cela va de soi dans la mesure où le possessif mon ne laisse pas de doute à ce sujet. Il est difficile, en effet, d'imaginer qu'il soit absent de son propre concert. L'engagement de l'énonciateur reste cependant entier, si nous modifions le co-texte pour donner à croire qu'il s'agit d'un disque et d'un concert assuré par une tierce personne :

(4") Je te donne son disque mais tu viens à son concert.

Les exemples (4) et (4'), ainsi que nos tentatives infructueuses de substitution par arriver, montrent que le recours au seul co-texte n'est pas suffisant, puisque l'identification du référent dépasse le cadre textuel. Il faudrait donc parler, dans ce cas, de contexte dissimulateur fort.

\section{2. ...dissimulateur faible}

L'exemple (5) et sa version (5') obtenue par substitution de venir par arriver, a fait l'objet d'un long débat ${ }^{(10)}$. Il est vrai que (5') est discutable à plus d'un égard :

(5) Message de A :

Bonjour

Je vais bientôt commencer mes travaux d'agrandissement et pour la dalle je compte faire sur un hérisson. Cependant où trouve-t-on ce genre de gros cailloux ? Merci.

(10) Atelier de travail du groupe « Lexique », ATILF-CNRS (UMR 7118), le 14 mars 2008. 
Message de B :

Va voir s'il n'y a pas de constructions dans ton coin, il y a souvent de beaux gros blocs et gros gravats! Les proprios sont contents de s'en séparer gratuitement.

Message de C :

Sinon tu viens chez moi, j'ai $10 \mathrm{~m} 3$ de gravats (béton, carrelage, tuile) à prendre gratuitement.

(5') Message de A :

Bonjour

Je vais bientôt commencer mes travaux d'agrandissement et pour la dalle je compte faire sur un hérisson. Cependant où trouve-t-on ce genre de gros cailloux ? Merci.

Message de B :

Va voir s'il n'y a pas de constructions dans ton coin, il y a souvent de beaux gros blocs et gros gravats! Les proprios sont contents de s'en séparer gratuitement.

Message de C :

Sinon tu arrives chez moi, j'ai $10 \mathrm{~m} 3$ de gravats (béton, carrelage, tuile) à prendre gratuitement.

D’un avis général, le message $\mathrm{C}$ de la version (5') fut considéré comme « moins bon » que la version originale. Il avait d'abord été rejeté, puisque contrairement à (5), il ne localisait pas les gravats, c'est-à-dire dissociait le lieu où ils se trouvaient de chez moi. Finalement, il a été jugé acceptable, mais uniquement à l'oral et sous réserve de disposer d'informations supplémentaires. De tels écarts de jugements ne peuvent qu'attirer davantage l'attention.

Les réserves émises à l'égard de la version (5') étaient, à chaque fois, liées aux problèmes de localisations, celle de gravats ou celle des protagonistes. Comparativement, venir dans (5) apparaît comme un élément fédérateur dans la construction de la signification du message $C$. Cet élément semble au contraire faire défaut dans la version $\left(5^{\prime} \mathrm{C}\right)$. Ce qui manque à (5'), à notre avis, c'est $1^{\prime}$ indication de la procédure à suivre pour identifier la personne de référence, comme cela se fait dans le cas de venir. Venir dans le message (5C) est accompagné d'un complément de lieu chez moi désignant le terminus du déplacement que doit faire figure. Il en est de même de sa version modifiée dans (5'). Originellement cependant, le message de Cdans (5) véhicule une indication supplémentaire : venir autorise une inférence au sujet de l'énonciateur. On comprend qu'au moment, quel qu'il soit, où figure $(t u)$ atteindra le terminus chez moi, l'énonciateur s'y trouvera également. Arriver de (5') reste neutre à cet égard. Le recouvrement des significations n'est donc que partiel, car il ne concerne que le déplacement de figure. Aussi bien dans (5) que dans (5'), il lui faudra parcourir la même trajectoire pour atteindre le terminus spécifié par chez moi. C'est par l'implication de l'énonciateur que les énoncés incriminés diffèrent. Contrairement à (5), (5') désengage l'énonciateur quant à son association au terminus du déplacement.

\section{3. ...assimilateurs forts}

Deux types de situations forment des co-textes dans lesquels la substitution entre venir et arriver débouche sur des énoncés pleinement acceptés et considérés comme synonymes. La substitution s'y opère donc salva significatione. L'analyse de notre corpus de travail a permis de distinguer deux types de configurations qui forment des co(n)-textes assimilateurs forts. Plus précisément, les co(n)-textes assimilateurs forts sont en grande partie tributaires du rôle assumé par je. 


\subsection{1. Énonciateur en tant que terminus}

Dans l'exemple (6), le long co-texte gauche met en place tous les éléments nécessaires pour l'interprétation de l'énoncé qui nous intéresse. Il constitue un scénario qui dispose tous les participants de la scène, et surtout il permet d'identifier, par une procédure anaphorique, le référent du pronominal $i l$, figure du déplacement, qui s'avère être le serpent, et non pas Yann, « ami des bêtes ». Comme le montre (6'), la substitution par arriver ne modifie pas la signification initiale de (6) :

(6) J'étais en train d'enjamber des arbres tombés, les autres étaient déjà passés et ne l'avaient pas remarqué.

Me retrouvant en équilibre sur un tronc, je le vois, je reste immobile, en criant : «Merde, un serpent! ».

Il était en train de se dénouer pour monter sur le même tronc que moi ! Le stress !!!

Dilemme : je m'éloigne vite fait, ou je fais comme on a appris et je fais l'arbre.

En plus, Yann, cet ami des bêtes, me disait « Vas-y, bouge pas, pour la photo et tout !»

Tu parles... Comme il venait vers moi, et que je n'étais pas sûr de reconnaître l'espèce, une fois qu'il était à $40 \mathrm{~cm}$ de ma cheville, je me suis barré ! (blog Internet)

(6') J'étais en train d'enjamber des arbres tombés, les autres étaient déjà passés et ne l'avaient pas remarqué.

Me retrouvant en équilibre sur un tronc, je le vois, je reste immobile, en criant : «Merde, un serpent! ».

Il était en train de se dénouer pour monter sur le même tronc que moi ! Le stress !!! Dilemme : je m'éloigne vite fait, ou je fais comme on a appris et je fais l'arbre. En plus, Yann, cet ami des bêtes, me disait « Vas-y, bouge pas, pour la photo et tout !» Tu parles... Commeil arrivait vers moi, et que je n'étais pas sûr de reconnaître l'espèce, une fois qu'il était à $40 \mathrm{~cm}$ de ma cheville, je me suis barré ! (blog Internet)

Dans (6) comme dans (6'), le locuteur, c'est-à-dire celui qui dit je, et l'énonciateur prenant en charge l'énoncé ne font qu'un. Il se constitue explicitement en «personne de référence ». C'est par son intermédiaire que nous identifions le terminus du déplacement par rapport auquel le mouvement de figure ( $i l$-serpent) est évalué. Plus simplement, le lieu «englobant» (11) l'énonciateur est en même temps le site du déplacement.

Le mécanisme de construction de la signification est cependant complexe, puisque l'énonciation en tant qu' acte individuel se fait dans un contexte qui efface son cadre spatio-temporel. Il s'agit, en effet, d'un écrit Internet qui remplit sa fonction communicative indépendamment de la situation spatio-temporelle de l'énonciateur au moment de son acte d'énonciation. Les caractéristiques spatio-temporelles de l'acte d'énonciation sont par ailleurs peu pertinentes pour le co-énonciateur, c'est-à-dire celui qui est en charge d'interpréter (6). En clair, il sait seulement que l'énonciateur était devant son ordinateur mais cette information est de peu d'utilité pour trouver le référent, en l'occurrence celui de il venait vers moi.

Jusque là, nous avons traité venir comme une expression token-réflexive, en adoptant la définition formulée par Kleiber (2008 : 124). Elle stipule que le référent d'une telle expression doit être trouvé, rappelons-le, «par l'intermédiaire d'éléments spatio-temporellement reliés à son occurrence ». Or, le seul lien spatio-temporel entre l'occurrence, qui relève de l'acte d'énonciation, et le référent, c'est-à-dire l'événement de mouvement, c'est l'énonciateur. En tant qu'individu, il est, certes, seul et unique, mais il peut aussi être appréhendé comme un ensemble d'instances spatio-tem-

(11) $C f$. Kleiber, 2008. 
porelles. Dans l'une d'entre elles, il a participé de l'événement décrit dans (6). C'est donc ainsi, en tant qu'ensemble instances spatio-temporelles, que l'énonciateur assure le lien entre l'événement et l'acte d'énonciation. Le contexte assimilateur fort de (6) est issu d'une configuration très particulière d'éléments sémantiques et discursifs : l'énonciateur, en tant qu'instance spatio-temporelle, est en même temps impliqué dans l'événement, puisque c'est lui qui permet d'identifier le point de référence spatiale par rapport auquel le mouvement s'effectue ${ }^{(12)}$.

\subsection{2. Énonciateur en tant que figure}

On remarque (Honeste, 2005) que lorsque le verbe venir est employé à la première personne, il perd son caractère déictique. Une perte de ce caractère consisterait donc à affranchir le processus de référence du recours aux éléments spatio-temporellement reliés à l'occurrence de ce verbe. L'exemple (7) permet de vérifier si un tel affranchissement a réellement lieu.

(7) Franchement, quand je viens à Lyon, je suis surpris par l'absence de bateauxmouches entre Rhône et Saône pour visiter le centre de Lyon jusqu'à l'Île Barbe. (Forum du Grand Lyon)

Comme dans l'exemple (6), le je est assumé, sur le plan discursif, à la fois par le locuteur et par l'énonciateur. Si nous abordons (7) du côté de son référent, c'est-àdire par l'événement de mouvement désigné ${ }^{(13)}$, leje représente figure de déplacement. Le terminus est explicitement identifié par le complément à Lyon. Les particularités sémantico-discursives d'une telle configuration ressortent clairement, lorsque 1'on compare (7) avec (4') :

(4") Je te donne son disque mais tu viens à son concert.

Dans les exemples (4") et (7), le terminus est explicitement spécifié, respectivement par à son concert et à Lyon. Comme nous l'avons vu dans la section 3.1., (4') est un contexte dissimulateur fort, ce qui n'est pas le cas de (7). En (7), la substitution se fait salva significatione:

(7') Franchement, quand j'arrive à Lyon, je suis surpris par l'absence de bateauxmouches entre Rhône et Saône pour visiter le centre de Lyon jusqu'à l' île Barbe.

La différence essentielle entre (4") et (7) réside dans le positionnement de l'énonciateur. En interprétant un énoncé tel que tu viens à son concert, c'est en vertu du choix de venir que nous associons l'énonciateur au terminus. Dans (7) l'énonciateur est en même temps figure de déplacement, il est donc supposé, au terme de celui-ci, rejoindre le terminus Lyon. Par conséquent, le mode d'association de l'énonciateur avec le terminus du déplacement tel que nous l'avons vu en (4) et en (4"') se trouve neutralisé par la sémantique du verbe venir.

Plutôt que de perte du caractère déictique par venir, nous préférons parler de sa neutralisation. Si, comme nous l'avons admis, c'est l'énonciateur qui constitue l'élément spatio-temporel nécessaire pour identifier le référent, il n'a pas disparu avec l'emploi de la première personne. Il a, au contraire, renforcé sa présence en tant que participant de l'événement de mouvement, en autorisant une relation synonymique forte avec arriver.

(12) Et, plus loin, l'exemple (8).

(13) Dans la mesure où cette information n'a pas d'incidence pour notre propos, nous ne faisons que signaler qu'il s'agit d'un événement pluriel. 


\section{4. ...assimilateur faible}

L'exemple (8) est intéressant, car le degré de synonymie entre arriver et venir en (8a) (8'a) n'est pas le même que celui qui relie venir et arriver dans la partie $(8 b)$ et $\left(8 b^{\prime}\right)$. En ce qui concerne ( $\left.8 \mathrm{a}\right)$ et ( 8 'a), la synonymie peut, à la suite de Duchacek, être considérée comme parfaite. Sans être explicitement spécifié par un complément, le site, lieu «pris comme référence », est accessible à travers l'énonciateur. Il s'agit donc d'une information déictique de type $1:$ le lieu de référence est identifié comme celui où se trouve l'énonciateur (qui l'englobe, dirait-on avec Kleiber). Pour ce qui est de (8a), il s'agit d'un co-texte assimilateur fort, comparable à celui de (6).

(8) Nous avons mangé notre jambon et nos compotes assis en tailleur devant l'exposition et il a dit : (8a) «Quand ils arrivent, les invités ? - (8b) Je ne crois pas qu'ils viendront ce soir », ai-je dit, le cœur arrêté. (Frantext)

(8') Nous avons mangé notre jambon et nos compotes assis en tailleur devant l'exposition et il a dit: ( $8^{\prime}$ a) «Quandils viennent, les invités ? - (8'b) Je ne crois pas qu'ils arriveront ce soir », ai-je dit, le cœur arrêté.

En ( $8 b)$ et ( $\left.8^{\prime} b\right)$, le lien de synonymie entre venir et arriver semble, en revanche, moins fort. C'est parce que, comme remarque Fuchs (1997: 37), « le type de co-texte ne peut se définir qu'à taille explicite. En effet, si l'on change la taille du co-texte, on peut en modifier le type : selon les cas, on ajoute ou on supprime des opérations, en sorte que la nature de l'interaction entre A (ou B) [A, B représentent les expressions synonymiques, en l'occurrence (8b) et (8'b) - DS ] et son environnement au sein de l'énoncé peut s'en trouver changée "».

Effectivement, pour arriver et venir, le co-texte n'est pas le même, selon qu'il s'agit de leurs occurrences en (8a) et (8'a) et celles qui apparaissent en (8b) et en $(8$ 'b). Même si $(8 a)$ et $(8 b)$ réfèrent à un seul et même événement de mouvement, (8b) se trouve dans la portée du modalisateur épistémique je ne crois pas. Ce modalisateur porte sur l'ensemble du contenu propositionnel ils viendront ce soir/ils arriveront ce soir. Le Querler (1996) qualifie une telle modalité d'extra-prédicative, traduisant une attitude extérieure au contenu propositionnel. Ce contenu propositionnel n'est pas identique. Il semblerait que le modalisateur actualise le sens lexical tel qu'il est défini en langue. Or, les défintions du TLFi présentent arriver et venir comme des quasi-synonymes : venir renvoie à un déplacement et peut être paraphrasé par aller à, alors qu'arriver sélectionne plutôt l'étape finale et la mise en contact avec le terminus. Nous y avons détecté une relation métonymique entre les sens lexicaux de arriver et venir, puisque l'étape finale fait bel et bien partie de la trajectoire, l'un des éléments conceptuels constitutifs d'un déplacement.

En ( $8 b$ ) et en ( $\left.8^{\prime} b\right)$, l'opérateur modal je ne crois pas que fait ressortir le sens propre à chacun des lexèmes. Par conséquent, avec venir l'énonciateur de (8b) met en doute le déplacement dans son intégralité. Il ne semble pas convaincu que les invités se soient tout simplement mis en route. Avec arriver dans (8'b), seul le fait d'atteindre le terminus est sujet à caution, d'autant plus que l'adverbial temporel ce soir localise cet événement dans le temps. Les invités (figure) ont bien entrepris le voyage, mais ils n'atteindront pas le terminus (nous) ce soir. Ainsi, pour ce qui est des relations synonymiques entre venir et arriver en (8b), l'exemple (8) représente-t-il un co-texte assimilateur faible où la commutation conduit à des énoncés «plus ou moins paraphrastiques, en ce sens que les valeurs prises par chacune des deux expressions A [venir dans l'énoncé (8b) de l'exemple (8) - DS] et B [arriver 
dans l'énoncé ( $8^{\prime}$ b) de l'exemple ( 8 'b) - DS] ne se chevauchent que partiellement» (Fuchs 1997:33)

\section{En guise de conclusion}

Accusée de tous les maux, mise en cause jusqu'à dans son existence (Honeste 2007), la synonymie est pourtant un fait linguistique, attestée par de nombreux exemples. Le cas de arriver et de venir nous paraît intéressant en ce qu'il montre toute la richesse du phénomène. Loin de l'avoir explorée complètement, nous en avons évoqué à peine quelques aspects. Autorisées en langue, les relations synonymiques entre arriver et venir ont un caractère dynamique en discours. Dans cette étude, nous avons essayé de déterminer le rôle de l'énonciateur qui constitue "l'élément spatio-temporel relié à l'occurrence » verbale. Notre corpus montre que les co(n)-textes assimilateurs sont ceux dans lesquels l'énonciateur adosse un double rôle sur le plan discursif et sémantique, en tant que participant du déplacement. Il suffit cependant d'un modalisateur, comme dans (8), pour affaiblir le lien de synonymie. Autant dire que la synonymie aura toujours de quoi surprendre...

\section{Bibliographie}

A Sher, N., SABlayrolles, P. (1995) : «A Typology and Discourse Semantics for Motion Verbs and Spatial PPs in French », Journal of Semantics, 12, 163-209.

A SNÈS, M., KUPFERMAN, L. (2008) : «Evénements, prédicats, arguments : quelques points de repère », Langages, 169, 7-33.

BoOnS, J.-P. (1985) : "Préliminaires à la classificationdes verbes locatifs : les compléments de lieu, leurs critères, leurs valeurs aspectuelles », Lingvisticae Investigationes IX, 2, 195-267.

- (1987) : «La notion sémantique de déplacement dans une classification syntaxique des verbes locatifs ", Langue française, 76, 5-40.

BOUCHARD, D. (1993) : «Primitifs, métaphore et grammaire : les divers emplois de venir et d'aller », Langue Française, 100, 49-66.

Bourdin, Ph. (1992) : «Constance et inconstance de la déicticité : la resémantisation des marqueurs andatifs et ventifs », La Deixis, Paris, Presses universitaires de France, 287-307.

— (2005) : «Venir en français contemporain : de deux fonctionnements périphrastiques », Le Querler, N. et Bat-Zeev Shyldkrot (éds), Les périphrases verbales. Lingvisticae Investigationes Supplementa, 25, 261-278.

Bouverot, D. (2007) : «La synonymie dans le TLF », Le français moderne, t. LXXV, 1, 7-14.

Cruse, A. (2004. Meaning in Language. An Introduction to Semantics and Pragmatics, Oxford University Press.

DUCHACEK, O. (1979) : "Synonymie en terminologie », Etudes romanes de Brno, $\mathrm{X}, 9-19$.

DUCROT, O., SCHAEFFER, J.-M. (1995) : Nouveau dictionnaire encyclopédique des sciences du langage, Paris, Editions du Seuil.

FuCHS, C. (1997) : «La synonymie en co-texte», Guimier,C. (éd.), Co-texte et calcul du sens, Presses universitaires de Caen, 31-41. 
HONESTE, M.-L. (2005) : «Venir est-il un verbe périphrastique ? Etude sémanticocognitive », Le Querler, N. et Bat-Zeev Shyldkrot (éds), Les périphrases verbales. Lingvisticae Investigationes Supplementa, 25, 295-310.

— (2007) : «Entre ressemblance et différence : synonymie et cognition », Le français moderne, t. LXXV, 1, 160-174.

KLEIBER, G. (2008) : «Comment fonctionne ICI», Vuillaume, M. (éd) Iciet maintenant. Cahiers Chronos, 20, 113-145.

LE QUERLER, N. (1996) : Typologie des modalités, Presses universitaires de Caen.

Neveu, F. (2004) : Dictionnaire des sciences du langage, Paris, Armand Colin.

TAlmy, L. (2000a) : Toward a Cognitive Semantics : Concept Structuring System. Volume I, Harvard, MIT Press.

- (2000b) : Toward a Cognitive Semantics : Typology and Process in Concept Structuring, Volume II, Harvard, MIT Press.

V ANDELOISE, C. (1987) : "La préposition à et le principe d'anticipation », Langue Française, 76, 77-111.

\section{Ressources électroniques}

DicoSyn/CRISCO, http ://www.cnrtl.fr/synonymie :

Trésor de la Langue Française informatisé, http ://atilf.atilf.fr/dendien

\section{PRATIQUES}

\section{PROCHAIN NUMÉRO}

$\mathrm{n}^{\circ} 143 / 144$ (décembre 2009)

\section{Écrits de savoir}

\section{Coordination : Mohamed Kara}

L'écriture des savoirs engage un certain nombre de difficultés que ce numéro voudrait contribuer à caractériser pour mieux les appréhender et, le cas échéant, les didactiser. Un enjeu des écrits des savoirs, et non des moindres, procède de la nécessité à laquelle sont tenus les scripteurs de construire et de conduire des parcours épistémologiques propres à ce que l'on pourrait appeler la codification écrite des savoirs. Si les soubassements épistémologiques paraissent si prégnants dans les écrits du savoir, c'est que le référentiel y occupe une place prépondérante, fût-il du langage, dans la mesure où il constitue l'objet premier de la recherche.C'est en premier lieu la connaissance d'un objet qui motive l'initiation d'une recherche et par suite sa formalisation écrite. A cet égard, l'enjeu aujourd'hui est autant de communiquer le résultat de ses recherches que les recherches elles-mêmes, bref l'on peut dire que la théorisation, comme le rappelle Neveu est aussi importante que la théorie. Or l'écriture des savoirs est une activité nécessairement alimentée, à l'université comme dans le secondaire et dans le premier degré, par d'autres activités (1'observation d'un objet ou d'un phénomène, les lectures d'information ou d'investigation, les interactions orales entre élèves et professeurs, les discussions entre pairs, à titre d'exemple) qui en sont du même coup constitutives puisqu'elles contribuent à la genèse de la pensée scripturale. C'est la raison principale pour laquelle ce numéro en préparation associe le travail de conception à celui de conceptualisation des écrits complexes. 